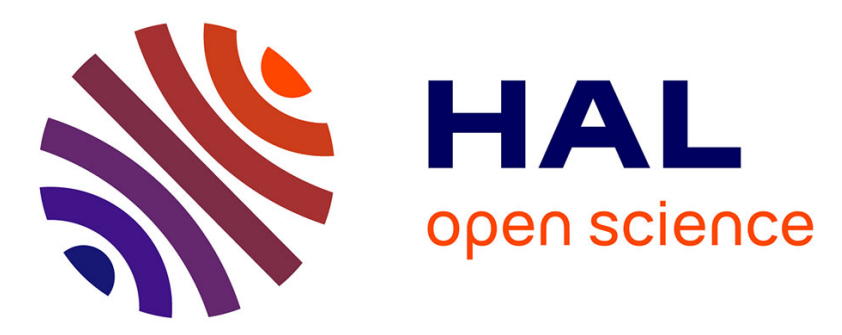

\title{
Polyharmonic distortion approach for nonlinear acoustic load characterization
}

\author{
M Volpe, S Bellizzi, Renaud Côte
}

\section{To cite this version:}

M Volpe, S Bellizzi, Renaud Côte. Polyharmonic distortion approach for nonlinear acoustic load characterization. Journal of Sound and Vibration, In press, 10.1016/j.jsv.2021.116426 . hal-03341408

\author{
HAL Id: hal-03341408 \\ https://hal.science/hal-03341408
}

Submitted on 10 Sep 2021

HAL is a multi-disciplinary open access archive for the deposit and dissemination of scientific research documents, whether they are published or not. The documents may come from teaching and research institutions in France or abroad, or from public or private research centers.
L'archive ouverte pluridisciplinaire HAL, est destinée au dépôt et à la diffusion de documents scientifiques de niveau recherche, publiés ou non, émanant des établissements d'enseignement et de recherche français ou étrangers, des laboratoires publics ou privés. 


\title{
Polyharmonic distortion approach for nonlinear acoustic load characterization
}

\author{
M. Volpe ${ }^{\mathrm{a}}$, S. Bellizzi ${ }^{\mathrm{a}}$, R. Côte ${ }^{\mathrm{a}}$ \\ ${ }^{a}$ Aix Marseille Univ, CNRS, Centrale Marseille, LMA \\ 4 impasse Nikola Tesla 13453 Marseille Cedex 13, FRANCE
}

\begin{abstract}
In this work, we analytically characterize the acoustic properties of a one-port nonlinear acoustic sample loaded on a waveguide. The classical reflection coefficient is extended as a scattering matrix relating the incident wave fundamental and harmonics amplitudes to the backward wave fundamental and harmonics amplitudes. Assuming that the acoustic load is defined as a nonlinear differential equation relating the acoustic pressure and the associated volume velocity, the scattering matrix is obtained by combining the harmonic balance method and the polyharmonic distortion approach. Theoretical and numerical aspects are considered. The proposed method is exemplified on two acoustic nonlinear absorbers.

Keywords: Scattering matrix, Nonlinear impedance, Polyharmonic distortion model, One-port nonlinear acoustic system, High Sound Level, Low Frequency
\end{abstract}

\section{Introduction}

The reduction of noise and vibration at low frequencies is still nowadays a main issue in many fields of engineering. In order to overpass this issue, a new concept of absorbers including nonlinear phenomena has been developed

5 in the past decade. This type of absorbers is based on the principle of the "Targeted Energy Transfer" (TET) also named "energy pumping" [1]. TET is an irreversible transfer of the vibrational energy from an input subsystem

Email address: cote@lma.cnrs-mrs.fr (R. Côte)

Preprint submitted to Journal of Sound and Vibration

August 10, 2021 
to a nonlinear attachment (the absorber) called Nonlinear Energy Sink (NES). TET permits to reduce undesirable large vibration amplitudes of structures or acoustic modes.

The basic NES generally consists of a lightmass, an essentially nonlinear spring and a viscous linear damper. In the field of structural vibration, a wide variety of NES designs with different types of stiffnesses (cubic [2], polynomial [3] non-polynomial [4, non-smooth nonlinearities [5]...) have been proposed.

15 In the acoustic field, it was demonstrated that a NES can be achieved by using a vibroacoustic coupling between the acoustic field (the primary system) and a geometrically nonlinear thin clamped structure (the NES). In [6, 7], the thin baffled structure consists of a simple thin circular latex (visco-elastic) membrane whereas in [8] a loudspeaker used as a suspended piston is considered. In both 20 cases, the thin baffled structure has to be part of the frontier of the closed acoustic domain (to be controlled). In [9], an hybrid electro-acoustic nonlinear membrane absorber working as a nonlinear energy sink is described. This NES is made of two elements: a membrane with a nonlinear dynamics described in [6, 7, and an active system based on a loudspeaker which controls the pressure applied to the rear face of the membrane. Finally in [10, it is shown that a Helmholtz resonator with a nonlinear behavior can be used as a NES to extract energy from one acoustical mode.

The experimental characterization of nonlinear devices remains a difficult task. Model-based or black box approaches can both be considered more generso ally as processes that characterize a device by a model and a set of parameters. For any device there is a balance to find between the model accuracy and the number of parameters, and the best balance depends on the application. This is why we are interested in refining and comparing characterization methods of nonlinear devices.

35 We have been developing a setup for the characterization of nonlinear acoustic absorbers as a one-port nonlinear acoustical system. A one-port nonlinear acoustical system is basically a system where there is a surface (the port) where acoustical interactions take place. In linear cases, the response can be given in 
terms of impedance or absorption coefficient. A review about linear impedance measurements can be found in Ref. [11. For nonlinear systems this approach is not suitable because the properties often change with the level of excitation, and can give rise to distortion (generation of harmonics). In the progress toward more accurate representations, we started with linearized characterizations (e.g. measurement of apparent reflection coefficients) used next for the identification 45 of nonlinear resonance parameters 12 .

In this paper, we advance one step forward. We use the extension of the concept of reflection coefficient toward multidimensional characterization thanks to the polyharmonic distortion approach. [13, 14, The main idea is to characterize the ability of a nonlinear element to generate harmonics, which can be treated in a second step as a source term in linear systems coupled to a nonlinear element. Instead of a single reflection coefficient, we use a pair of scattering matrices describing the interactions between harmonics. The principle of energy transfer between harmonics is a well-known approach for the characterization of nonlinear systems [15] but to the best of our knowledge it has not been generalized with scattering matrices.

Our results hold for systems which dynamics is described by differential equations in time domain.

The paper is organized as follows. The first part describes the class of systems under study: one-port nonlinear acoustical systems. In the second part we establish analytically the diffusion matrix which characterizes how the acoustical system sends back an incident tonal acoustic wave, in the form of a superposition of harmonics. The analytic formulation is derived from the combination of the harmonic balance method and the polyharmonic distortion method which permits a fully analytic calculation of the scattering matrices. In the third part we apply our results on two examples of nonlinear devices, and we check the consistency of the results with a direct approach based on numerical time integration of the equations of motion. 


\section{System under study}

In this paper, all quantities in the frequency domain, unless otherwise stated, will be considered assuming a $e^{+\mathrm{j} 2 \pi f t}$ temporal dependence.

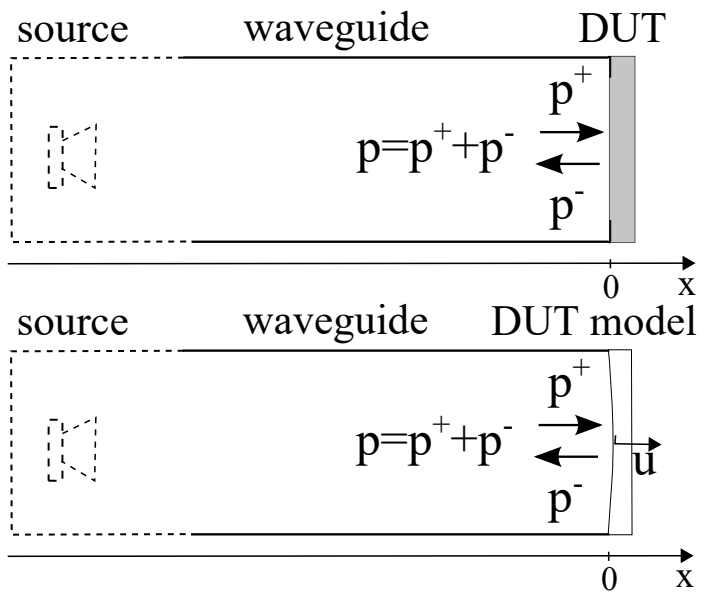

Figure 1: Schematic representation of the system under study: (a) DUT connected to a cylindrical waveguide, (b) equivalent DUT model connected to a cylindrical waveguide.

Our system, see Fig. 1(a), consists of a Device Under Test (DUT) connected to one side of a wave-guide and submitted to an acoustic field resulting from a source located at the opposite side of the wave guide. The waveguide is a cylindrical duct of cross-section $S$. In this work, the acoustic source and the 75 propagation in the waveguide, considered as planed waves (one-dimensional), are assumed linear. The acoustic pressure at $x=0$, i.e the acoustic pressure in front of the DUT inside the waveguide, can be written as

$$
p(t)=p^{+}(t)+p^{-}(t)
$$

where $p^{+}$(respectively $p^{-}$) denotes the incoming (respectively backward) acoustic wave with respect the DUT. When the DUT is linear (Helmholtz absorber case for example), it can be characterized in the frequency domain by the reflection coefficient function defined as

$$
R(f)=\frac{P^{-}(f)}{P^{+}(f)}
$$


where $P^{+}$(respectively $P^{-}$) denotes the Fourier transform of $p^{+}$(respectively $\left.p^{-}\right)$.

From now on, we assume that the DUT that we will consider in this paper ${ }_{85}$ can be modelled (see Fig. 1(b)) as

$$
m_{1} \ddot{u}(t)+c_{1} \dot{u}(t)+k_{1} u(t)+g_{\mathrm{nl}}(u(t), \dot{u}(t))=S_{\mathrm{e}} p(t)
$$

where $u$ denotes the equivalent transversal displacement of the outer surface of the DUT and the dot represents time differentiation. The first three parameters $m_{1}, c_{1}$ and $k_{1}$ characterize the linear part of the DUT (Helmholtz absorber case for example) and the nonlinear function $g_{\mathrm{nl}}$ with respect to $u(t)$ and $\dot{u}(t)$ the

90 nonlinear part. $S_{\mathrm{e}}$ denotes the effective cross-section of the DUT $\left(S_{\mathrm{e}}\right.$ can be smaller than $S$ the cross-section of the waveguide). Note that only the acoustic pressure in front on the membrane inside the waveguide is used in Eq. (3) and the acoustic pressure outside the waveguide is neglected.

Following the assumption on the acoustic propagation, the outer surface of ${ }_{95}$ the DUT can be related to the incoming and backward acoustic pressures, and to the associated volume velocity $q$ as

$$
\begin{aligned}
\dot{u}(t) & =\frac{1}{\rho_{\mathrm{a}} c_{\mathrm{a}}}\left(p^{+}(t)-p^{-}(t)\right) \\
q(t) & =S_{\mathrm{e}} \dot{u}(t)
\end{aligned}
$$

where $\rho_{\mathrm{a}}$ is the density of air and $c_{\mathrm{a}}$ the sound velocity in air.

Considering only the linear part of Eq. (3) and substituting Eqs. (1), (4) and (5) into the resulting Eq. (3) written in the frequency domain, we get the reflection coefficient of the underlying linear oscillator, acting here like a tuned mass damper :

$$
R(f)=\frac{\mathrm{j}(2 \pi f) m_{\mathrm{l}}+c_{\mathrm{l}}+\frac{k_{\mathrm{l}}}{\mathrm{j}(2 \pi f)}-\rho_{\mathrm{a}} c_{\mathrm{a}} S_{\mathrm{e}}}{\mathrm{j}(2 \pi f) m_{\mathrm{l}}+c_{\mathrm{l}}+\frac{k_{\mathrm{l}}}{\mathrm{j}(2 \pi f)}+\rho_{\mathrm{a}} c_{\mathrm{a}} S_{\mathrm{e}}} .
$$




\section{Scattering matrix representation in a nonlinear DUT}

To deepen the description of the acoustic load when nonlinearities are present, the linear impedance model has to be extended to take into account nonlinear

\subsection{Principle}

From now on we assume that the acoustic source generates an excitation at only the excitation frequency $f$ (the fundamental frequency) and we are interested in the associated periodic responses with period $T=1 / f$.

The resulting acoustic pressure in front of the DUT and the associated incoming and backward pressures can be written as a Fourier series in complex exponential form truncated to order $H$ as

$$
\begin{gathered}
p(t)=\sum_{k=-H}^{H} P_{k}(f) e^{\mathrm{j} 2 \pi k f t} \\
p^{+}(t)=\sum_{k=-H}^{H} P_{k}^{+}(f) e^{\mathrm{j} 2 \pi k f t} \text { and } p^{-}(t)=\sum_{k=-H}^{H} P_{k}^{-}(f) e^{\mathrm{j} 2 \pi k f t}
\end{gathered}
$$

where $P_{k}(f), P_{k}^{+}(f)$ and $P_{k}^{-}(f)$ denote the Fourier coefficients of order $k$ also named $k^{\text {th }}$ harmonics satisfying $P_{-k}(f)=\overline{P_{k}(f)}, P_{-k}^{+}(f)=\overline{P_{k}^{+}(f)}$ and $P_{-k}^{-}(f)=$ $\overline{P_{k}^{-}(f)}$ where $\overline{(.)}$ denotes complex conjugate. The harmonic terms have to be taken into account due to the nonlinear behavior of the DUT. We have chosen to indicate the frequency dependency of the Fourier coefficients using the fundamental frequency $f$. However, it is important to recall that, the $k^{\text {th }}$ harmonics are related to the frequency $k f$, as indicated by the exponential terms.

The scattering matrix can be viewed as an extension of the reflection coefficient concept. It characterizes the relationship between the harmonic terms $\left(P_{k}^{+}(f), 0 \leq k \leq H\right)$ of the incoming acoustic pressure and the harmonic terms $\left(P_{k}^{-}(f), 0 \leq k \leq H\right)$ of the backward acoustic pressure as

$$
P_{k}^{-}(f)=\sum_{n=0}^{H} S_{k n}(f) P_{n}^{+}(f) \text { for } k=0,1, \cdots, H
$$


or in matrix from

$$
\mathbf{P}^{-}(f)=\mathbf{S}(f) \mathbf{P}^{+}(f)
$$

where

$$
\begin{aligned}
& \mathbf{P}^{+}(f)=\left(P_{0}^{+}(f), P_{1}^{+}(f), \cdots, P_{H}^{+}(f)\right)^{T}, \\
& \mathbf{P}^{-}(f)=\left(P_{0}^{-}(f), P_{1}^{-}(f), \cdots, P_{H}^{-}(f)\right)^{T}
\end{aligned}
$$

and $\mathbf{S}(f)=\left(S_{k n}(f)\right)_{0 \leq k \leq H, 0 \leq n \leq H}$ denotes the scattering matrix at the frequency $f$. The component $S_{k n}(f)$ represents the transfer of energy from the harmonic term, $P_{n}^{+}(f)$, of the incoming acoustic pressure (at the frequency ${ }_{130} n f$ ) to the harmonic term, $P_{k}^{-}(f)$, of the backward acoustic pressure (at the frequency $k f)$.

When the DUT is linear, it is easy to show that $\mathbf{S}(f)$ is a diagonal matrix satisfying

$$
S_{k k}(f)=R(k f) \text { for } k=0,1, \cdots, H
$$

where $R$ denotes the reflection coefficient of the DUT as defined in Eq. (2). Note that as expected $\mathbf{S}$ does not depend on the involved energy level.

When the DUT is nonlinear, obviously $\mathbf{S}(f)$ will be non diagonal and will depend on the involved energy level.

\subsection{Theoretical approach}

Our objective is to develop a method to build analytically the scattering matrix. The method is developed from the dynamic model of the DUT (Eq. 3) and combines harmonic balance method [16] to solve Eq. (3) and polyharmonic distortion modeling [13] to obtain the scattering matrix.

\subsubsection{Harmonic balance method}

The Harmonic Balance Method (HBM) is one of the most interesting methods to obtain the periodic solutions of the non-linear dynamical systems as Eq. (3). We represent the harmonic excitation $p(t)$ and the DUT response $u(t)$ 
as a Fourier series described up to the $H^{\text {th }}$ term as

$$
\begin{aligned}
& p(t)=\sum_{k=-H}^{H} P_{k}(f) e^{\mathrm{j} 2 \pi k f t} \\
& u(t)=\sum_{k=-H}^{H} U_{k}(f) e^{\mathrm{j} 2 \pi k f t}
\end{aligned}
$$

where $P_{-k}(f)=\overline{P_{k}(f)}$ and $U_{-k}(f)=\overline{U_{k}(f)}$.

150 frequency up to the $H^{\text {th }}$ term, one obtains a nonlinear algebraic system in $\mathbb{C}$ of $H+1$ equations which can be written as

$$
\tilde{\mathbf{\mathbf { R }}}(f, \mathbf{U}(f), \mathbf{P}(f))=\mathbf{Z}(f) \mathbf{U}(f)+\mathbf{G}_{\mathrm{nl}}(\mathbf{U}(f))-S_{\mathrm{e}} \mathbf{P}(f)=0
$$

where

$$
\begin{aligned}
& \mathbf{U}(f)=\left(U_{0}(f), U_{1}(f), \cdots, U_{H}(f)\right)^{T} \in \mathbb{C}^{H+1}, \\
& \mathbf{P}(f)=\left(P_{0}(f), P_{1}(f), \cdots, P_{H}(f)\right)^{T} \in \mathbb{C}^{H+1},
\end{aligned}
$$

$\mathbf{Z}(f)$ is a diagonal matrix which diagonal elements are

$$
\left(k_{1}, k_{1}-(2 \pi f)^{2} m_{1}+\mathrm{j}(2 \pi f) c_{1}, \cdots, k_{1}-(2 \pi k f)^{2} m_{1}+\mathrm{j}(2 \pi k f) c_{1}, \cdots\right)
$$

155 for $k=1, \cdots, H$ and

$$
\mathbf{G}_{\mathrm{nl}}(\mathbf{U}(f))=\left(G_{\mathrm{nl}_{0}}(\mathbf{U}(f)), G_{\mathrm{nl}_{1}}(\mathbf{U}(f)), \cdots, G_{\mathrm{nl}_{H}}(\mathbf{U}(f))\right)^{T}
$$

is the vector of nonlinear effects defined as

$$
g_{\mathrm{nl}}(u(t), \dot{u}(t))=\sum_{k=-H}^{H} G_{\mathrm{nl}_{k}}(\mathbf{U}(f)) e^{\mathrm{j} 2 \pi k f t} .
$$

where $G_{\mathrm{nl}_{k}}(\mathbf{U}(f))$ denotes the $k^{\text {th }}$ harmonics of the time function $g_{\mathrm{nl}}(u(t), \dot{u}(t))$.

If $g_{\mathrm{nl}}$ is a polynomial in both $u$ and $\dot{u}$, the coefficients $G_{\mathrm{nl}_{k}}(\mathbf{U}(f))$ can be obtained exactly using a recursive method at least for low-order polyno- 
Frequency-Time approach has to be used [16. This case will not be considered here. It results that in this paper, $\tilde{\tilde{\mathbf{R}}}$ is polynomial with respect to the components of $\mathbf{U}(f)$.

It is easy to realize that for a tonal (single frequency) excitation, the nonlinear function generates a system response distributed over several harmonics. Depending on the choices of nonlinear function, excitation frequency and cut-off order, the response can display higher harmonics (also called superharmonics), lower harmonics (subharmonics) or both [1].

Introducing the velocity term $v(t)=\dot{u}(t)$ as a new variable, Eq. 16) can be rewritten with respect to the Fourier coefficients, $V_{k}(f)$ for $k=1, \cdots, H\left(V_{0}\right.$ is always equal to zero), of $v(t)$ defined by

$$
U_{k}(f)=\frac{V_{k}(f)}{\mathrm{j} k(2 \pi f)}
$$

and the zero-order harmonic $U_{0}$ of $u(t)$ as

$$
\tilde{\mathbf{R}}(f, \mathbf{V} \mathbf{V}(f), \mathbf{P}(f))=\tilde{\tilde{\mathbf{R}}}(f, \boldsymbol{\Lambda}(f) \mathbf{V} \mathbf{V}(f), \mathbf{P}(f))=0
$$

where

$$
\mathbf{V V}(f)=\left(U_{0}, V_{1}(f), V_{2}(f), \cdots, V_{H}(f)\right)^{T} \in \mathbb{C}^{H+1}
$$

and $\boldsymbol{\Lambda}(f)$ is a diagonal matrix which diagonal elements are

$$
\left(1, \frac{1}{\mathrm{j} 2 \pi f}, \frac{1}{\mathrm{j} 2 \pi 2 f}, \cdots, \frac{1}{\mathrm{j} 2 \pi H f}\right) .
$$

Note that the first component of the vector $\mathbf{V V}(f)$ is defined as the zero-order harmonic of $u(t)$ and other components to the non-zero-order harmonic of $v(t)$.

Finally considering (1) and (4) written in frequency domain, the Fourier coefficients of $p(t)$ are related to the coefficients of $p^{+}(t)$ and $p^{-}(t)$ as

$$
P_{k}(f)=P_{k}^{+}(f)+P_{k}^{-}(f) \quad \text { for } k=0,1, \cdots, H
$$

and the Fourier coefficients of $v(t)$ are related to the coefficients of $p^{+}(t)$ and $p^{-}(t)$ as

$$
V_{k}(f)=\frac{1}{\rho_{\mathrm{a}} c_{\mathrm{a}}}\left(P_{k}^{+}(f)-P_{k}^{-}(f)\right) \quad \text { for } k=1,2, \cdots, H
$$


with the complementary property

$$
P_{0}^{-}(f)=P_{0}^{+}(f)
$$

The relation (28) means that the incoming and backward acoustic pressure waves have equal zero-order harmonic coefficient which are equal the half values of the zero-order harmonic coefficient of $p(t)$. It comes from the hypothesis that the mean velocity is null, i.e. that there is no flow in the system.

Substituting Eqs. (26), (27) and (28) into Eq. (23), one obtains a new nonlinear algebraic system in $\mathbb{C}$ of $H+1$ equations as

$$
\mathbf{R}\left(f, \mathbf{P}^{+}(f), \mathbf{P} \mathbf{P}^{-}(f)\right)=\tilde{\mathbf{R}}\left(f, \frac{1}{\rho_{\mathrm{a}} c_{\mathrm{a}}}\left(\mathbf{P}^{+}(f)-\mathbf{P}^{-}(f)\right)+\mathbf{U}_{0}(f), \mathbf{P}^{+}(f)+\mathbf{P}^{-}(f)\right)=0
$$

where $\mathbf{P}^{+}(f)$ and $\mathbf{P}^{-}(f)$ are defined by Eqs. (11) and 12 and

$$
\begin{aligned}
\mathbf{P P}^{-}(f) & =\left(U_{0}(f), P_{1}^{-}(f), P_{2}^{-}(f), \cdots, P_{H}^{-}(f)\right)^{T} \in \mathbb{C}^{H+1} \\
\mathbf{U}_{0}(f) & =\left(U_{0}(f), 0, \cdots, 0\right)^{T} \in \mathbb{C}^{H+1}
\end{aligned}
$$

Note that the first component of the vector $\mathbf{P P}^{-}(f)$ is defined as the zero-order harmonic of $u(t)$ and other components to the non-zero-order harmonic of $p^{-}(t)$.

System (29) is a family of implicit functions which characterize the DUT by correlating the harmonic (or spectral) components, $P_{k}^{+}(f)$, of the incoming acoustic pressure with the components $P_{k}^{-}(f)$ of the backward acoustic pressure and the zero-order harmonic of $u(t)$. The scattering matrix definition, Eq. 107, appears as a "linear" approximation of the system $(29)$ considered as a relationship between $\mathbf{P}^{+}(f)$ and $\mathbf{P} \mathbf{P}^{-}(f)$. The linearization step will be considered in the next section.

\subsubsection{Polyharmonic distortion modeling}

In this section the frequency dependency will be omitted.

There exist different techniques of linearization. The most used in the context of nonlinear dynamical system is based on a minimization procedure related to the difference between the nonlinear system and the linear one. However, this 
class of methods needs an averaging operator to define a well posed problem. For example, in the context of experimentation, the averaging operator is built from a family of measured points characterizing the resulting linearization procedure as a global approximation. In this work we focus on a local approach as described in [13], named PolyHarmonic Distortion (PHD) modeling, relying on Taylor expansion.

The PHD modeling is based on two assumptions: (i) the system is a timeinvariant system and (ii) the fundamental component $P_{1}^{+}$of the incoming acoustic pressure $p^{+}(t)$ is dominant (i.e $\left|P_{k}^{+}\right|<<\left|P_{1}^{+}\right|$for all $k>1$ ).

From assumption (i), if $\left(\mathbf{P}^{+}, \mathbf{P} \mathbf{P}^{-}\right)$satisfies Eq. 29 then it is easy to show that the couple $\left(\mathbf{P}_{\text {mod }}^{+}, \mathbf{P} \mathbf{P}_{\text {mod }}^{-}\right)$, defined by

$$
\begin{aligned}
\mathbf{P}_{\bmod }^{+} & =\left(P_{0}^{+},\left|P_{1}^{+}\right|, P_{2}^{+} \Theta_{1}^{-2}, \cdots, P_{H}^{+} \Theta_{1}^{-H}\right)^{T}, \\
\mathbf{P P}_{\bmod }^{-} & =\left(U_{0}, P_{1}^{-} \Theta_{1}^{-1}, P_{2}^{-} \Theta_{1}^{-2}, \cdots, P_{H}^{-} \Theta_{1}^{-H}\right)^{T}
\end{aligned}
$$

where $\Theta_{1}$ is chosen as

$$
\Theta_{1}=e^{\mathrm{j} \varphi_{1}} \text { with } P_{1}^{+}=\left|P_{1}^{+}\right| e^{\mathrm{j} \varphi_{1}},
$$

215

satisfies also Eq. (29) i.e

$$
\mathbf{R}\left(\mathbf{P}_{\bmod }^{+}, \mathbf{P P}_{\bmod }^{-}\right)=0
$$

Introducing the vector $\mathbf{P}_{\mathbf{0}}{ }^{+}$as

$$
\mathbf{P}_{\mathbf{0}}^{+}=\left(0,\left|P_{1}^{+}\right|, 0, \cdots, 0\right)^{T}
$$

which only depends on the modulus of the fundamental component of $\mathbf{P}^{+}, \mathbf{P}_{\bmod }^{+}$ can be written as

$$
\mathbf{P}_{\bmod }^{+}=\mathbf{P}_{\mathbf{0}}^{+}+\tilde{\mathbf{P}}^{+}
$$

where

$$
\tilde{\mathbf{P}}^{+}=\left(P_{0}^{+}, 0, P_{2}^{+} \Theta_{1}^{-2}, \cdots, P_{H}^{+} \Theta_{1}^{-H}\right)^{T} .
$$

220 Now from assumption (ii), we have

$$
\left\|\tilde{\mathbf{P}}^{+}\right\|<<\left\|\mathbf{P}_{\mathbf{0}}^{+}\right\|
$$


suggesting that $\mathbf{P P}_{\text {mod }}^{-}$can be approximated performing a first-order Taylor expansion around $\mathbf{P}_{\mathbf{0}}{ }^{+}$solving

$$
\mathbf{R}\left(\mathbf{P}_{0}{ }^{+}, \mathbf{P P}_{0}{ }^{-}\right)=\mathbf{0}
$$

with respect to $\mathbf{P P}_{\mathbf{0}}{ }^{-}$. These properties will be used later on.

The first step is to re-write the nonlinear algebraic system (35) with respect 225 to real variables as

$$
\mathbf{G}\left(\left|P_{1}^{+}\right|, \mathbf{X}, \mathbf{Y}\right)=\mathbf{0}
$$

where $\mathbf{G}$ is a $(2 H+1)$-dimensional vector function (different from $\left.G_{\mathrm{nl}}\right), \mathbf{G}=$ $(\operatorname{Re}(\mathbf{R}), \operatorname{Im}(\mathbf{R}))^{T}$ with $\operatorname{Re}($.$) (respectively \operatorname{Im}($.$) ) denotes real (respectively imag-$ inary) part, $\mathbf{X} \in \mathbb{R}^{2(H-1)+1}$ with

$$
\mathbf{X}=\left(P_{0}^{+}, \operatorname{Re}\left(P_{2}^{+} \Theta_{1}^{-2}\right), \cdots, \operatorname{Re}\left(P_{H}^{+} \Theta_{1}^{-H}\right), \operatorname{Im}\left(P_{2}^{+} \Theta_{1}^{-2}\right), \cdots, \operatorname{Im}\left(P_{H}^{+} \Theta_{1}^{-H}\right)\right)^{T}
$$

and $\mathbf{Y} \in \mathbb{R}^{2 H+1}$ with

$$
\mathbf{Y}=\left(U_{0}, \operatorname{Re}\left(P_{1}^{-} \Theta_{1}^{-1}\right), \cdots, \operatorname{Re}\left(P_{H}^{-} \Theta_{1}^{-H}\right), \operatorname{Im}\left(P_{1}^{-} \Theta_{1}^{-1}\right), \cdots, \operatorname{Im}\left(P_{H}^{-} \Theta_{1}^{-H}\right)\right)^{T}
$$

230 The vector $\mathbf{X}$ does not depend on the fundamental component $P_{1}^{+}$of $p^{+}(t)$ but of course the function $\mathbf{G}$ depends directly on the modulus of $P_{1}^{+}$.

It is easy to verify that solving Eq. (40) with respect to $\mathbf{P} \mathbf{P}_{\mathbf{0}}{ }^{-}$is equivalent to solve

$$
\mathbf{G}\left(\left|P_{1}^{+}\right|, 0, \mathbf{Y}\right)=\mathbf{0}
$$

with respect to $\mathbf{Y}$.

235 Following the implicit function theorem, Eq. 41. can be converted to

$$
\mathbf{Y}=\mathbf{F}\left(\left|P_{1}^{+}\right|, \mathbf{X}\right)
$$

where $\mathbf{F}$ is a $(2 H+1)$-dimensional vector function of the real variables $\mathbf{X}$ with Jacobian matrix,

$$
\mathbf{J}_{\mathbf{F}}(\mathbf{X})=\left(\frac{\partial \mathbf{F}_{i}}{\partial \mathbf{X}_{\mathbf{j}}}(\mathbf{X})\right)_{1 \leq i \leq 2 H+1,1 \leq \mathrm{j} \leq 2(H-1)+1},
$$


given by

$$
\mathbf{J}_{\mathbf{F}}(\mathbf{X})=\mathbf{J}_{\mathbf{G}}^{\mathbf{Y}}\left(\mathbf{X}, \mathbf{F}\left(\left|P_{1}^{+}\right|, \mathbf{X}\right)\right)^{-1} \mathbf{J}_{\mathbf{G}}^{\mathbf{X}}\left(\mathbf{X}, \mathbf{F}\left(\left|P_{1}^{+}\right|, \mathbf{X}\right)\right)
$$

where $\mathbf{J}_{\mathbf{G}}^{\mathbf{X}}$ (respectively $\mathbf{J}_{\mathbf{G}}^{\mathbf{Y}}$ ) denotes the Jacobian matrix of $\mathbf{G}$ with respect to ${ }_{240} \mathbf{X}$ (respectively $\left.\mathbf{Y}\right)$.

Applying the Taylor expansion of order 1 to the function $\mathbf{F}$ around the null vector $\mathbf{X}=\mathbf{0}$, Eq. 45 reduce to

$$
\left.\mathbf{F}\left(\left|P_{1}^{+}\right|, \mathbf{X}\right)\right) \approx \mathbf{F}\left(\left|P_{1}^{+}\right|, \mathbf{0}\right)+\mathbf{J}_{\mathbf{F}}(\mathbf{0}) \mathbf{X}
$$

which can be used to approximate $\mathbf{Y}$.

Finally the last step consists to rewrite Eq. 48, with respect to the complex variables $\mathbf{P}^{+}$and $\mathbf{P} \mathbf{P}^{-}$using Eqs. 42 and 43 and the following relations

$$
\begin{aligned}
\operatorname{Re}\left(P_{k}^{+} \Theta_{1}^{-k}\right) & =\frac{1}{2}\left(P_{k}^{+} \Theta_{1}^{-k}+\overline{\left(P_{k}^{+} \Theta_{1}^{-k}\right)}\right) \\
\operatorname{Im}\left(P_{k}^{+} \Theta_{1}^{-k}\right) & =\frac{1}{2 \mathrm{j}}\left(P_{k}^{+} \Theta_{1}^{-k}-\overline{\left(P_{k}^{+} \Theta_{1}^{-k}\right)}\right) .
\end{aligned}
$$

for $k=1, \cdots, H$.

After some algebraic manipulations, the zero-order harmonic of $u(t)$ can be expressed as

$$
U_{0}=\sum_{n=0}^{H} S 0_{n}\left(\left|P_{1}^{+}\right|, \Theta_{1}\right) P_{n}^{+}+\sum_{n=0}^{H} T 0_{n}\left(\left|P_{1}^{+}\right|, \Theta_{1}\right) \overline{P_{n}^{+}}
$$

where

$$
\begin{aligned}
S 0_{0} & =\mathbf{J}_{\mathbf{F}}(0)_{1,1} \\
S 0_{1} & =\frac{\mathbf{F}_{1}\left(\left|P_{1}^{+}\right|, 0\right)}{\left|P_{1}^{+}\right|} \Theta_{1}^{n-1} \\
S 0_{k} & =\frac{1}{2}\left(\mathbf{J}_{\mathbf{F}}(0)_{1, k}+\frac{1}{\mathrm{j}} \mathbf{J}_{\mathbf{F}}(0)_{1, k+H-1}\right) \Theta_{1}^{-k}, \quad \text { for } k=2, \cdots, H \\
T 0_{0} & =0 \\
T 0_{1} & =0 \\
T 0_{k} & =\frac{1}{2}\left(\mathbf{J}_{\mathbf{F}}(0)_{1, k}-\frac{1}{\mathrm{j}} \mathbf{J}_{\mathbf{F}}(0)_{1, k+H-1}\right) \Theta_{1}^{k}, \quad \text { for } k=2, \cdots, H
\end{aligned}
$$

and the harmonic coefficients of $p^{-}(t)$ can be expressed with respect to the harmonic coefficients of $p^{+}(t)$ as

$$
P_{k}^{-}=\sum_{n=0}^{H} S_{k, n}\left(\left|P_{1}^{+}\right|, \Theta_{1}\right) P_{n}^{+}+\sum_{n=0}^{H} T_{k, n}\left(\left|P_{1}^{+}\right|, \Theta_{1}\right) \overline{P_{n}^{+}}
$$


or in matrix form

$$
\mathbf{P}^{-}=\mathbf{S}\left(\left|P_{1}^{+}\right|, \Theta_{1}\right) \mathbf{P}^{+}+\mathbf{T}\left(\left|P_{1}^{+}\right|, \Theta_{1}\right) \overline{\mathbf{P}^{+}}
$$

where the components of the complex matrices $\mathbf{S}\left(\left|P_{1}^{+}\right|, \Theta_{1}\right)$ and $\mathbf{T}\left(\left|P_{1}^{+}\right|, \Theta_{1}\right)$ are given by

- for $n=0$

$$
\begin{aligned}
& S_{0,0}=1 \\
& S_{0, k}=0, \quad \text { for } k=1, \cdots, H \\
& T_{0, k}=0, \quad \text { for } k=0, \cdots, H
\end{aligned}
$$

- for $n=1, \cdots, H$,

$$
\begin{aligned}
S_{n, 0} & =\left(\mathbf{J}_{\mathbf{F}}(0)_{n+1,1}+\mathbf{j} \mathbf{J}_{\mathbf{F}}(0)_{H+n+1,1}\right) \Theta_{1}^{n} \\
S_{n, 1} & =\frac{\mathbf{F}_{n+1}\left(\left|P_{1}^{+}\right|, 0\right)+\mathbf{j} \mathbf{F}_{H+n+1}\left(\left|P_{1}^{+}\right|, 0\right)}{\left|P_{1}^{+}\right|} \Theta_{1}^{n-1} \\
S_{n, k} & =\frac{1}{2}\left[\left(\mathbf{J}_{\mathbf{F}}(0)_{n+1, k}+\mathbf{J}_{\mathbf{F}}(0)_{H+n+1, H+k-1}\right)\right. \\
& \left.+\mathbf{j}\left(\mathbf{J}_{\mathbf{F}}(0)_{H+n+1, k}-\mathbf{J}_{\mathbf{F}}(0)_{n+1, H+k-1}\right)\right] \Theta_{1}^{n-k}, \quad \text { for } k=2, \cdots, H \\
T_{n, 0} & =0 \\
T_{n, 1} & =0 \\
T_{n, k} & =\frac{1}{2}\left[\left(\mathbf{J}_{\mathbf{F}}(0)_{n+1, k}-\mathbf{J}_{\mathbf{F}}(0)_{H+n+1, H+k-1}\right)\right. \\
& \left.+\mathbf{j}\left(\mathbf{J}_{\mathbf{F}}(0)_{H+n+1, k}+\mathbf{J}_{\mathbf{F}}(0)_{n+1, H+k-1}\right)\right] \Theta_{1}^{n+k}, \quad \text { for } k=2, \cdots, H .
\end{aligned}
$$

Equation (54) can also be expressed as a single matrix relationship

$$
\mathbf{P}^{\prime-}=\mathbf{S}^{\prime}\left(\left|P_{1}^{+}\right|, \Theta_{1}\right) \mathbf{P}^{\prime+}
$$

where

$$
\begin{aligned}
& \mathbf{P}^{\prime+}(f)=\left(P_{-H}^{+}(f), \cdots, P_{-1}^{+}(f), P_{0}^{+}(f), P_{1}^{+}(f), \cdots, P_{H}^{+}(f)\right)^{T}, \\
& \mathbf{P}^{\prime-}(f)=\left(\left(P_{-H}^{-}(f), \cdots, P_{-1}^{-}(f), P_{0}^{-}(f), P_{1}^{-}(f), \cdots, P_{H}^{-}(f)\right)^{T} .\right.
\end{aligned}
$$

\subsubsection{Some properties of $\mathbf{S}$ and $\mathbf{T}$}

From Eq. (54), the vector $\mathbf{P}^{-}$appears as a sum of two terms, the first one is linear with respect to $\mathbf{P}^{+}$whereas the second one is only $\mathbb{R}$-linear with respect 
to $\mathbf{P}^{+}$. It is easy to verify that the modulus of the components of the matrices $\mathbf{S}$ and $\mathbf{T}$ only depend on the modulus of the fundamental component, $P_{1}^{+}$.

Moreover, the first column of $\mathbf{S}$ only depends on the function $\mathbf{F}$. This column characterizes the transfer of energy from the fundamental component $P_{1}^{+}$ of $p^{+}(t)$ (assuming dominant in this approximation) to all the harmonic components of $\mathbf{P}^{-}$. The other columns of $\mathbf{S}$ and the matrix $\mathbf{T}$ only depend on the Jacobian matrix $\mathbf{J}_{\mathbf{F}}(0)$ which corresponds to corrective terms in the first-order Taylor expansion.

If the DUT is linear $\left(\mathbf{G}_{\mathrm{nl}}\right.$ is zero), it is easy to show from Eq. 29 that all the terms $\mathbf{T}_{n, k}$ are equal to zero. Hence, Eq. (54) takes the same form as Eq. (10). Moreover, the matrix $\mathbf{S}$ is diagonal and satisfies the same relation as Eq. 13.

In the nonlinear case, Eq. (54) generally differs from Eq. 10 except if the 275 function $\mathbf{F}$ as a complex function is holomorphic (i.e. satisfies the CauchyRieman condition) at $\mathbf{X}=0$ which signify that

$$
\begin{aligned}
\mathbf{J}_{\mathbf{F}}(0)_{n+1, k} & =\mathbf{J}_{\mathbf{F}}(0)_{H+n+1, H+k-1} \\
\mathbf{J}_{\mathbf{F}}(0)_{n+1, H+k-1} & =-\mathbf{J}_{\mathbf{F}}(0)_{H+n+1, k}
\end{aligned}
$$

resulting to $T_{n, k}=0$. In the cases where the matrix $\mathbf{T}$ differs from the zero matrix, it will be interesting to evaluate the respective influence of $\mathbf{S}$ and $\mathbf{T}$ to reproduce $\mathbf{P}^{-}$.

\subsection{Associated numerical procedure}

In practice, a numerical procedure is required to obtain the decomposition (54). For a given model (3), a given harmonic number $H$ and a given acoustic pressure $p(t)$ defined by its $H+1$ harmonic coefficients in a frequency band $\left[f_{\min }, f_{\max }\right]$, the proposed procedure includes the following steps:

- Step 1: Eq. 237 is first re-written in the real domain as

$$
\tilde{\mathbf{R}}^{\mathrm{RI}}\left(f, \mathbf{V} \mathbf{V}^{\mathrm{RI}}, \mathbf{P}^{\mathrm{RI}}\right)=0,
$$


where the real function $\tilde{\mathbf{R}}^{\mathrm{RI}}$ is defined as

$$
\tilde{\mathbf{R}}^{\mathrm{RI}}=\left(\tilde{\mathbf{R}}_{1}, \operatorname{Re}\left(\tilde{\mathbf{R}}_{2}\right), \cdots, \operatorname{Re}\left(\tilde{\mathbf{R}}_{H+1}\right), \operatorname{Im}\left(\tilde{\mathbf{R}}_{2}\right), \cdots, \operatorname{Im}\left(\tilde{\mathbf{R}}_{H+1}\right)\right),
$$

the real vector $\mathbf{V} \mathbf{V}^{\mathrm{RI}}$ as

$\mathbf{V} \mathbf{V}^{\mathrm{RI}}=\left(\mathbf{V} \mathbf{V}_{1}, \operatorname{Re}\left(\mathbf{V} \mathbf{V}_{2}\right), \cdots, \operatorname{Re}\left(\mathbf{V} \mathbf{V}_{H+1}\right), \operatorname{Im}\left(\mathbf{V} \mathbf{V}_{2}\right), \cdots, \operatorname{Im}\left(\mathbf{V} \mathbf{V}_{H+1}\right)\right)$

and the real vector $\mathbf{P}^{\mathrm{RI}}$ as

$$
\mathbf{P}^{\mathrm{RI}}=\left(P_{0}, \operatorname{Re}\left(P_{1}\right), \cdots, \operatorname{Re}\left(P_{H}\right), \operatorname{Im}\left(P_{1}\right), \cdots, \operatorname{Im}\left(P_{H}\right)\right) .
$$

Assuming $\mathbf{P}^{\mathrm{RI}}$ known, Eq. 62 is solved in the frequency band $\left[f_{\min }, f_{\max }\right]$

\section{Application}

This section is devoted to illustrating the scattering matrix representation introduced in Section 3. To this end, two nonlinear absorbers (NES) are con-

- Step 2: A partition $f_{i}$ of $\left[f_{\min }, f_{\max }\right]$ with $f_{i}=f_{\min }+i \Delta f$ for $i=$ $0, \cdots, N_{f}$ and $\Delta f=\left(f_{\max }-f_{\min }\right) / N_{f}$ is considered. For each frequency $f_{i}$,

1. $\mathbf{P}^{+}\left(f_{i}\right)$ and $\mathbf{P}^{-}\left(f_{i}\right)$ are computed from Eqs.(1) and (4) written in Fourier series using the couple solution $(\mathbf{V V}, \mathbf{P})$ of Eq. 62 at $f=f_{i}$.

2. From $\mathbf{P}^{+}\left(f_{i}\right)$, Eq. (44) is solved with respect to $\mathbf{Y}$ (using the function FindRoot of (c)Mathematica) and

3. The jacobian matrix of $\mathbf{F}$ is obtained from Eq. 47) with $\mathbf{X}=0$ and $\mathbf{F}\left(\left|P_{1}^{+}\right|, 0\right)=\mathbf{Y}$.

4. Finally, the matrices $\mathbf{S}$ and $\mathbf{T}$ are deduced from Eqs. (55) and (56). sidered. 


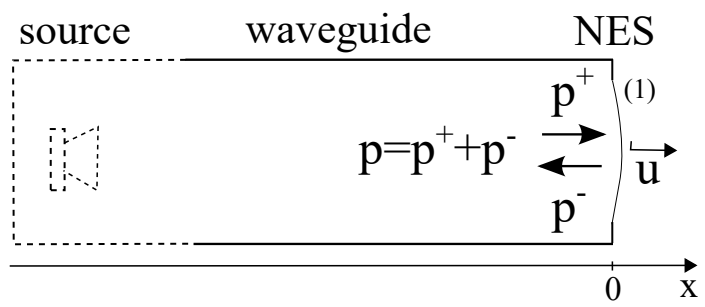

Figure 2: Schematic representation of the NES membrane: (1) circular visco-elastic membrane.

\subsection{NES membrane}

In this first example, a cylindrical pre-stressed clamped nonlinear viscoelastic membrane with radius $R_{\mathrm{m}}$, cross-section $S_{\mathrm{m}}$ and thickness $h_{\mathrm{m}}$ is considered (see Fig. 22). The nonlinear behavior results from geometrical nonlinearity when large displacement of the membrane occur. This acoustic load has been introduced in [7] as NES and can be modelled as a simplified model of the membrane motion formulated as a one DOF nonlinear oscillator

$$
m_{\mathrm{m}} \ddot{u}(t)+c_{\mathrm{m}} \dot{u}(t)+k_{\mathrm{m}} u(t)+k_{3 \mathrm{~m}}\left(u(t)^{3}+2 \eta u(t)^{2} \dot{u}(t)\right)=\frac{S_{\mathrm{m}}}{2} p(t)
$$

where the dot represents time differentiation and $u$ denotes the transversal displacement of the centre of the membrane. Note that only the acoustic pressure in front on the membrane inside the waveguide is used in Eq. 66 and the acoustic pressure outside the waveguide is neglected .

The numerical values of the parameters used in this study as deduced in Appendix B are given by $m_{\mathrm{m}}=0.000265 \mathrm{~kg}, c_{\mathrm{m}}=0.0001 \mathrm{~N} \mathrm{~m}^{-1}, k_{\mathrm{m}}=$ $10.5 \mathrm{~N} \mathrm{~m}^{-1}, k_{3 \mathrm{~m}}=2.646 \times 10^{6} \mathrm{~N} \mathrm{~m}^{-3}, \eta=0.00114$ and $S_{\mathrm{m}}=\pi 0.03^{2} \mathrm{~m}^{2}$.

Taking advantage of the symmetries of the problem, a numerical study has been conducted, considering only odd harmonics. The corresponding developments are not given here.

In this numerical study, we assume that the acoustic pressure $p(t)$ is periodic as

$$
p(t)=P_{-1} e^{-\mathrm{j} 2 \pi f t}+P_{1} e^{\mathrm{j} 2 \pi f} .
$$




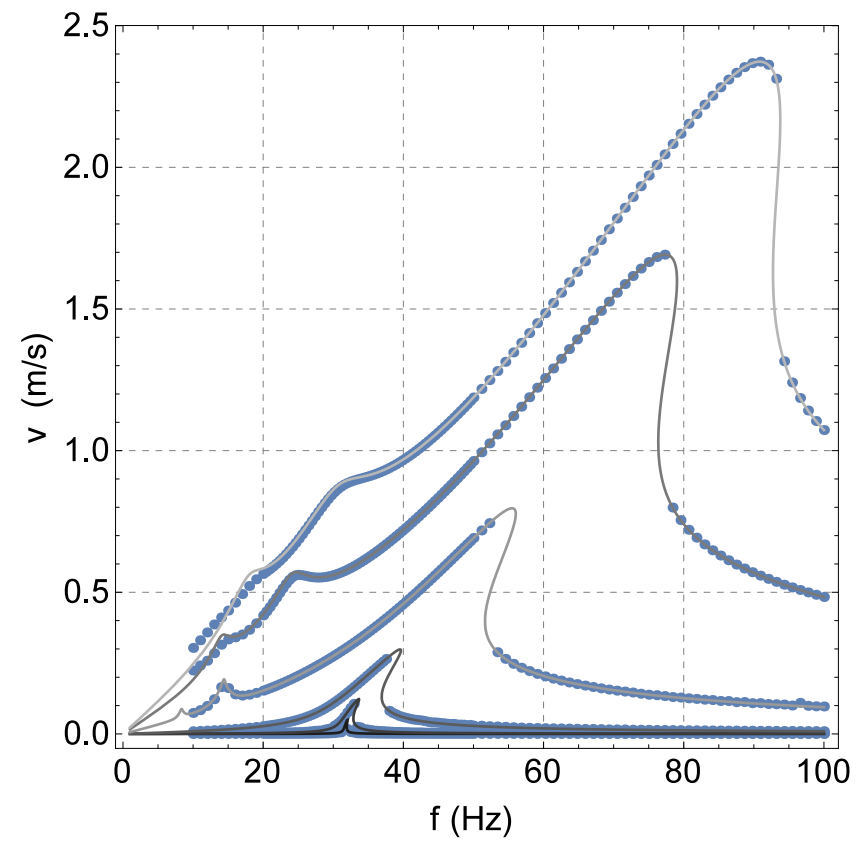

Figure 3: Numerical simulation of Eq. 66 in time domain (blue dot markers) and using HBM (Eq 23p)with six levels of the fundamental component of $p(t):\left|P_{1}\right|=0.01,0.1,1,10,50$ and 100. (displayed in gray level, from bottom to top).

Figure 3 shows the RMS values of the steady-state response $v(t)$ of Eq. (66) obtained by HBM using only the first three odd harmonics (harmonics 1, 3 and 5 ) solving the corresponding nonlinear system $(62)$ as described Section 3.3 (Step 1). The excitation frequency $f$ was chosen from $1 \mathrm{~Hz}$ to $100 \mathrm{~Hz}$. Six excitation levels were used: $\left|P_{1}\right|=0.01,0.1,1,10,50$ and 100 . corresponding to 53.9 to $133.9 \mathrm{~dB}$. Also plotted are the responses obtained from the nonlinear equations of motion (66) using the ordinary differential equations solver NDSolve (with the choice Automatic for the option Method) available in (c) Mathematica. The excitation frequency $f$ was chosen from $10 \mathrm{~Hz}$ to $100 \mathrm{~Hz}$ with a frequency step of $1 \mathrm{~Hz}$. HBM approach gives results in very good agreement, independently of the excitation level, with the direct solving approach considered here as the exact solution. This comparison validates the number of harmonics used in this numerical study. 

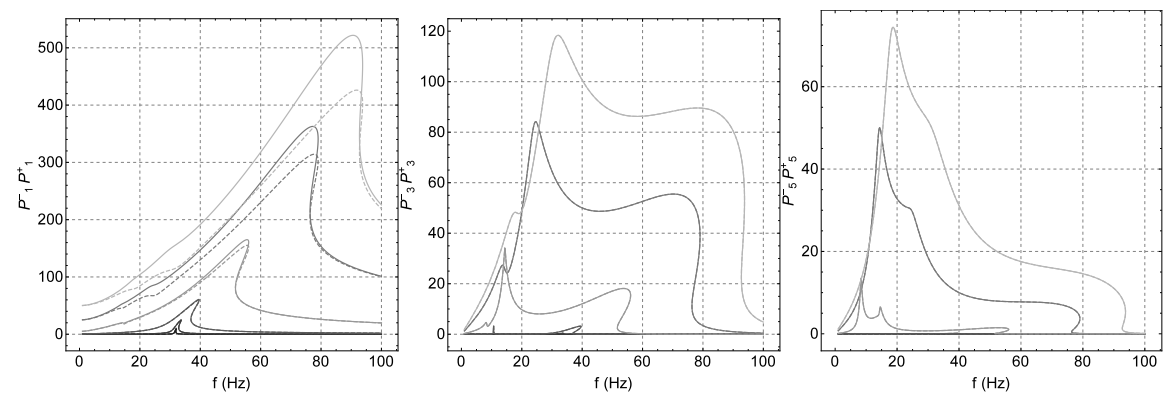

Figure 4: Modulus of the harmonic components of $p^{+}(t)$ (continuous lines) and $p^{-}(t)$ (dashed lines) obtained from HBM approach six levels of the fundamental component of $p(t):\left|P_{1}\right|=$ $0.01,0.1,1,10,50$ and 100 . (displayed in gray level from bottom to top).

Figure 4 shows the corresponding harmonic coefficients $P_{1}^{+}, P_{3}^{+}$and $P_{5}^{+}$ (respectively $P_{1}^{-}, P_{3}^{-}$and $P_{5}^{-}$) of $p^{+}(t)$ (respectively $p^{-}(t)$ ) obtained from HBM as described Section 3.3 (Step 2). We can see that as expected $\left|P_{1}^{+}(f)\right| \geq$ $\left|P_{1}^{-}(f)\right|$ and that the difference increases with the excitation level. Moreover for $k>1$, and due to the fact that the high harmonic $P_{k}(f)$ of $p(t)$ are equal to zero, we have $P_{k}^{+}(f)=-P_{k}^{-}(f)$.

The components of the matrix $\mathbf{S}$ are reported Fig. 5 . As expected the diagonal terms of $\mathbf{S}$ decrease whereas the non diagonal terms of $\mathbf{S}$ increase with the excitation level. Instead, the diagonal term $S_{n, n}$ represents the ratio of energy between the $n$th harmonic of $p^{+}(t)$ and the $n$th harmonic of $p^{-}(t)$ whereas the term $S_{k, n}$ represents the ratio of energy transmitted from the $n$th harmonic of $p^{+}(t)$ to the $k$ th harmonic of $p^{-}(t)$. The square of the terms of $\mathbf{S}$ is homogeneous to ratios of power transfers (in $\mathrm{W} / \mathrm{W}$ ) between harmonics.

For a given excitation level, the behavior of the diagonal terms of $\mathbf{S}$ are very similar and are strongly affected by the nonlinear behavior of the NES when the excitation level increases. The scaling transformation as described by Eq. 13p is here masked. It is only visible at low excitation levels.

The first column of $\mathbf{S}$ corresponds to the energy transfer of the fundamental component $P_{1}^{+}$of $p^{+}(t)$ to the harmonic $P_{k}^{-}$(for $k=1,3$ and 5 ) of $p^{-}(t)$. We deduce that when the level increases the participation of $P_{1}^{+}$to $P_{1}^{-}$is reduced 

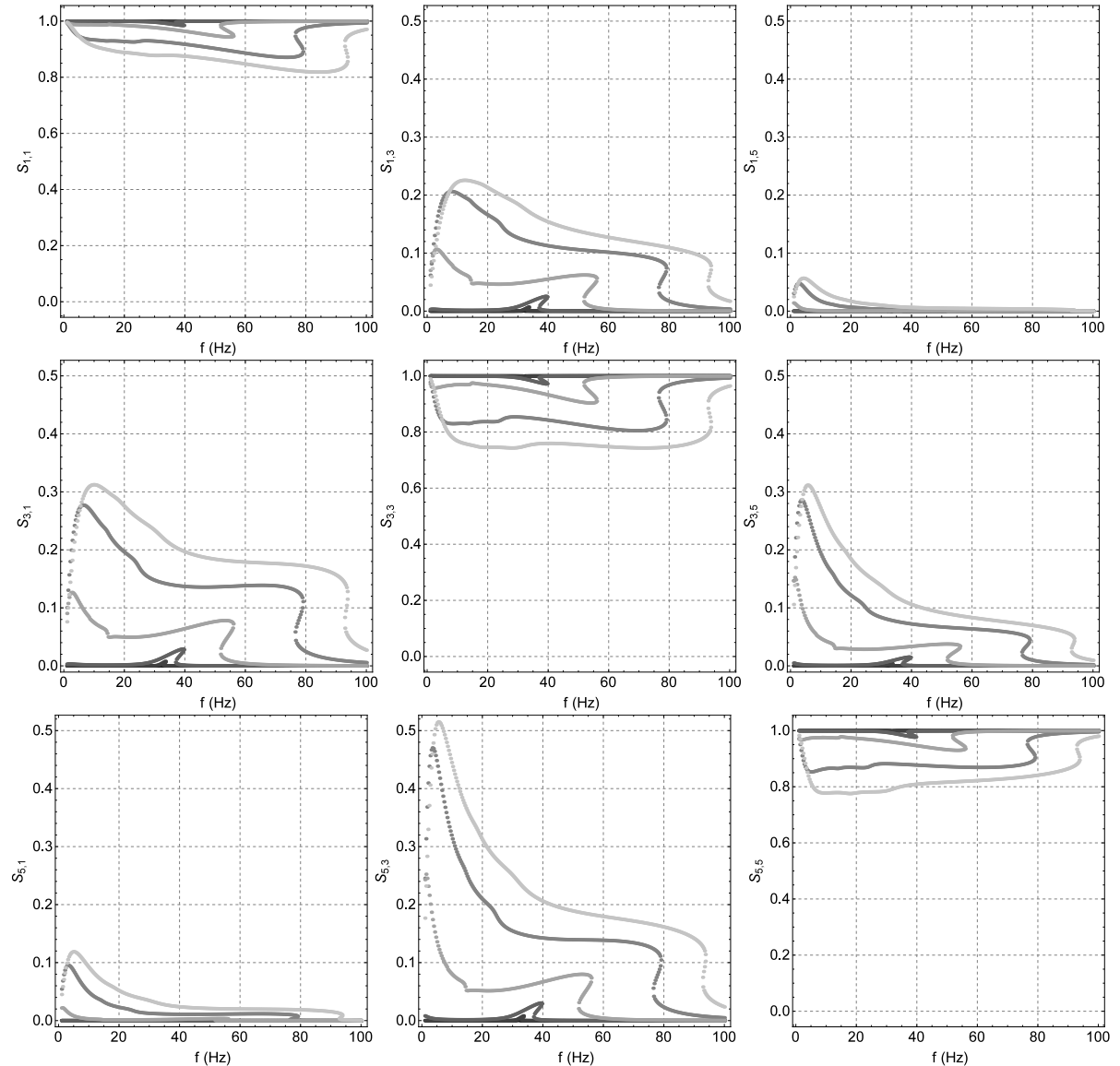

Figure 5: Modulus of the components of the matrix $\mathbf{S}$ obtained with the procedure described Section 3.3 for six levels of the fundamental component of $p(t):\left|P_{1}\right|=0.01,0.1,1,10,50$, and 100 (displayed in gray level from bottom to top).

whereas the participation of $P_{1}^{+}$to $P_{k}^{-}$for $k>1$ is amplified. Moreover the amplification decreases when the $k$ increase. Note that the frequency band where these phenomena are visible increases also with the excitation level. The same phenomenon can be observed on the second (respectively third) column of $\mathbf{S}$. These columns correspond to the energy transfer of the 3th (respectively 5th) harmonic of $p^{+}(t)$ to harmonic $P_{k}^{-}$(for $k=1,3$ and 5) of $p^{-}(t)$.

The components of the matrix $\mathbf{T}$ is reported in Fig. 6. In contrast to $\mathbf{S}$, all components of $\mathbf{T}$ are small and only affected by the nonlinear behavior at low 

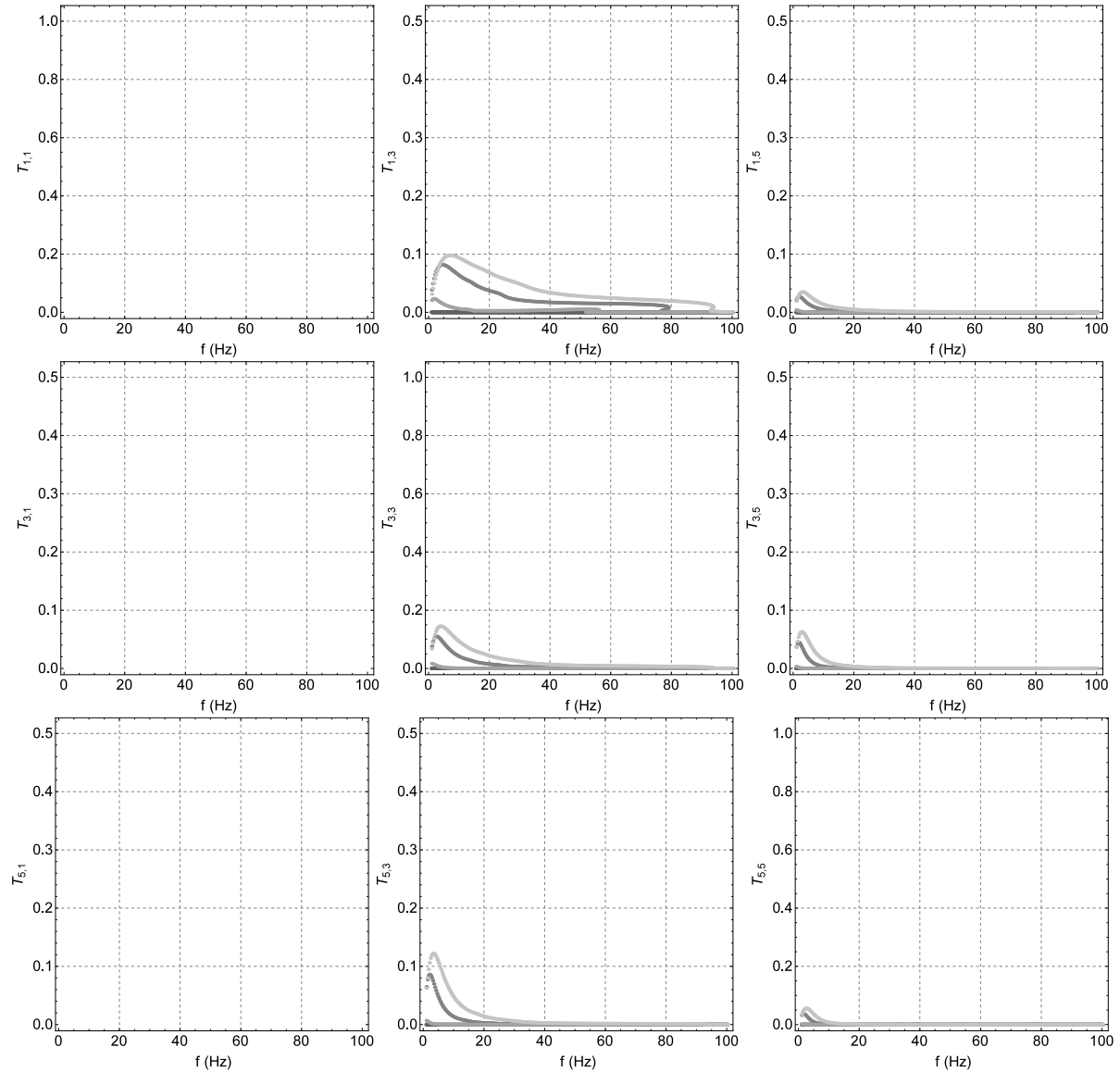

Figure 6: Modulus of the components of the matrix $\mathbf{T}$ obtained with the procedure described Section 3.3 for six levels of the fundamental component of $p(t):\left|P_{1}\right|=0.01,0.1,1,10$ and 100 (displayed in gray level from bottom to top).

frequencies $(<20 \mathrm{~Hz})$ showing that $\overline{\mathbf{P}^{+}}$does not affect significantly $\mathbf{P}^{-}$.

The last point to analyse is the efficiency of Eq. (54) as prediction formula of $\mathbf{P}^{-}$from $\mathbf{P}^{+}$. Figure 7 compares the modulus of $p_{1}^{-}, p_{3}^{-}$and $p_{5}^{-}$obtained from the HBM approach and using Eq. (54) taking into account only the matrix S. Only two levels of excitation are considered : the smaller and the higher. In both cases, Eq. (54) gives results in good agreement with the HBM approach. These results confirm that the procedure captures correctly the influence of the nonlinear elements of the NES membrane. We have also reported on Fig. 7, the 

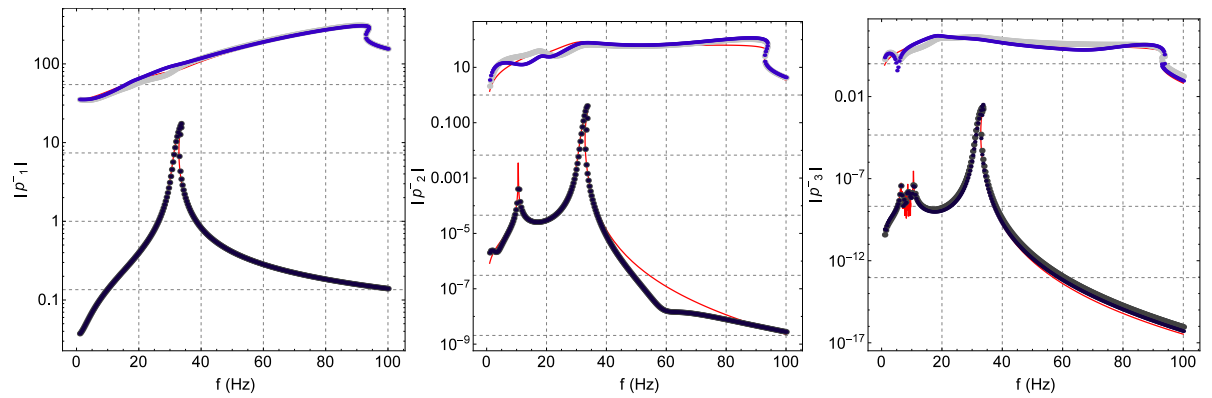

Figure 7: Modulus of the harmonic components of $p^{-}(t)$ obtained with the HBM (continuous red lines) and with the polyharmonic distortion decomposition Eq. 54 taking into account only the matrix $\mathbf{S}$ (grey and black dots) and taking into account only the reduced matrix $S$ (blue dots) for two levels: $\left|P_{1}\right|=0.1$ and 100 .

prediction of $\mathbf{P}^{-}$obtained from Eq. (54) neglecting the matrix $\mathbf{T}$ and reducing $\mathbf{S}$ to this first column and to the linear part of the diagonal terms. To be more specific, $S_{3,3}$ and $S_{5,5}$ were obtained from Eq. (56) using the part of the Jacobian $\mathbf{J}_{\mathbf{F}}(0)$ due to the linear part of $\mathbf{F}$. The results show a good agreement with both the HBM approach and the prediction including all the structure of $\mathbf{S}$.

The membrane NES is a good candidate to an experimental validation of the theory developed in this article, because its dynamical model is well established.

\subsection{Nonlinear Helmholtz resonator}

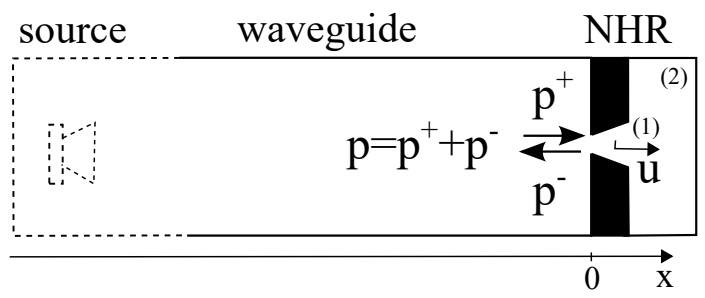

Figure 8: Schematic representation of the NHR: (1) tailored neck and (2) cavity.

This second example is dedicated to a Nonlinear Helmholtz Resonator (NHR) as introduced in [17] and detailed in [18. The NHR consists (see Fig. 8) in an air column in the tailored cylindrical neck considered as an incompressible mass and 
the air inside the cylindrical cavity which is supposed to be compressible. The friction due to the air at high velocity in the neck produces damping whereas the compression of the air in the cavity produces a restoring force. Hence, nonlinear terms take place in damping and restoring forces.

As shown in [18, 17, the NHR can be modelled as a one DOF nonlinear oscillator written here with dimensionless variables as

$$
\ddot{u}(t)+\delta \dot{u}(t)+\sigma|\dot{u}(t)| \dot{u}(t)+u(t)-\alpha u(t)^{2}+\beta u(t)^{3}=s p(t)
$$

with $u=\frac{S}{V_{0}} U$ and $t=\omega_{0} \bar{t}$ where $U$ is the displacement of the air in the neck, $V_{0}$ is the volume of the NHR cavity and $S$ is the cross section of the neck, $\bar{t}$ is the real time and $\omega_{0}$ is the resonance angular frequency of the NHR in the linear regime. The quadratic nonlinear damping term is due to the jet phenomenon and the nonlinear restoring force involves a quadratic term and a cubic term originating essentially from the air enclosed in the cavity of a resonator.

The numerical values of the parameters of $(68)$ used in this study are given by $\delta=0.005, \sigma=0.05, \alpha=1.2, \beta=1.36$ and $s=0.001$. These numerical values are associated to a NHR with a cavity length $0.0215 \mathrm{~m}$ and section $0.0066 \mathrm{~m}^{2}$ and with a tailed neck length $0.009 \mathrm{~m}$, external radius $0.002 \mathrm{~m}$ and internal radius $0.004 \mathrm{~m}$. The resulting natural resonance angular frequency of the NHR in the linear regime is $\omega_{0}=2 \pi 246 \mathrm{rad} / \mathrm{s}$.

Like in the previous example, we assume that the acoustic pressure $p(t)$ is periodic as

$$
p(t)=P_{-1} e^{-\mathrm{j} 2 \pi f t}+P_{1} e^{\mathrm{j} 2 \pi f} .
$$

Figure 9 shows the RMS values of the steady-state response $v(t)=\dot{u}(t)$ of Eq. (68) obtained by HBM with $H=3$ corresponding to harmonics 0,1 , 2 and 3 solving the nonlinear system $(62)$ as described Section 3.3 (Step 1). The excitation frequency $f$ was chosen from $0.142 \mathrm{~Hz}$ to $0.164 \mathrm{~Hz}$. Six excitation amplitudes were used: $\left|P_{1}\right|=0.071,1.61,3.15,4.69,6.24$ and $7.78 \mathrm{~Pa}$ corresponding to 71 to $112 \mathrm{~dB}$. Also plotted are the responses obtained from the nonlinear equations of motion (68) using the ordinary differential equations solver NDSolve (with the choice Automatic for the option Method) available in 


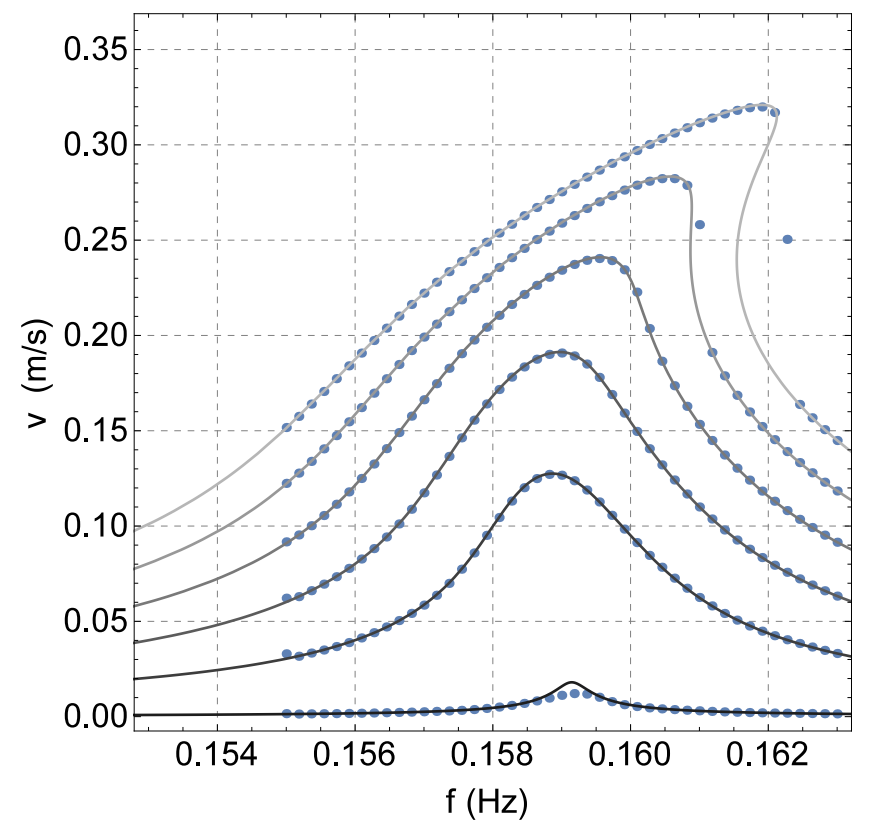

Figure 9: Numerical simulation of Eq. 68 in time domain (blue dot markers) and using HBM (Eq 23) with six levels of the fundamental component of $\left|P_{1}\right|=0.071,1.61,3.15,4.69,6.24$ and 7.78 (displayed in gray level from bottom to top).

(c) Mathematica. The excitation frequency $f$ was chosen from $0.155 \mathrm{~Hz}$ to 0.164 Hz.HBM approach gives results in very good agreement, independently of the excitation level, with the direct solving approach considered here as the exact solution. This comparison validates the number $H=3$ of harmonics used in this numerical study. These curves reveal the nonlinear softening and hardening behavior of the HNR in nonlinear regimes.

Figure 10 shows the corresponding harmonic coefficients $P_{1}^{+}, P_{2}^{+}$and $P_{3}^{+}$ (respectively $P_{1}^{-}, P_{2}^{-}$and $P_{3}^{-}$) of $p^{+}(t)$ (respectively $p^{-}(t)$ ) obtained from HBM as described Section 3.3 (Step 2). We can see that as expected $\left|P_{1}^{+}(f)\right| \geq$ $\left|P_{1}^{-}(f)\right|$ and that the difference increases with the excitation level. Moreover for $k>1$, and due to the fact that the high harmonic $P_{k}(f)$ of $p(t)$ are equal to zero, we have $P_{k}^{+}(f)=-P_{k}^{-}(f)$. It is interesting to note that the nonlinear softening and hardening behavior is also well visible. 

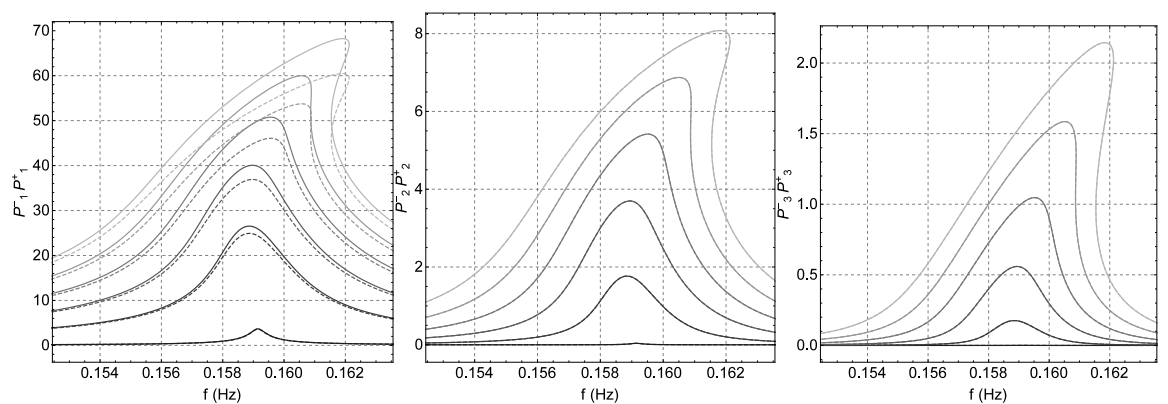

Figure 10: Modulus of the harmonic components of $p^{+}(t)$ (continuous lines) and $p^{-}(t)$ (dashed lines) obtained from HBM approach six levels of the fundamental component of $\left|P_{1}\right|=0.071,1.61,3.15,4.69,6.24$ and 7.78 (displayed in gray level from bottom to top).

The components of the matrix $\mathbf{S}$ are reported Fig. 11. Many behaviors observed in these plots match those of the early example.

More specifically, the diagonal terms of $\mathbf{S}$ decrease whereas the non diagonal terms of $\mathbf{S}$ increase with the excitation level. We deduce that when the level from the HBM approach and using Eq. (54) taking into account only the matrix S. Only two levels of excitation are considered, the smaller and the higher. In both cases, Eq. 54 gives results in good agreement with the HBM approach. These results confirm that the procedure captures correctly the influence of the increases the participation of $P_{k}^{+}$to $P_{k}^{-}$is reduced whereas the participation of $P_{n}^{+}$to $P_{k}^{-}$for $k \neq n$ is amplified.

For a given excitation level, the behavior of the diagonal terms of $\mathbf{S}$ are very similar and are strongly affected by the nonlinear behavior of the NES in a narrow frequency band when the excitation level increases. The scaling transformation as described by Eq. (13) is only visible at low excitation level.

The components of the matrix $\mathbf{T}$ is reported in Fig. 12 In contrast to $\mathbf{S}$, all components of $\mathbf{T}$ are small and only affected by the nonlinear behavior in a narrow frequency band showing that $\overline{\mathbf{P}^{+}}$does not affect significantly $\mathbf{P}^{-}$.

The last point to analyse is the efficiency of Eq. (54) as prediction formula of $\mathbf{P}^{-}$from $\mathbf{P}^{+}$. Figure 13 compares the modulus of $p_{1}^{-}, p_{2}^{-}$and $p_{3}^{-}$obtained nonlinear elements of the NES.We have also reported on Fig. 7, the prediction 

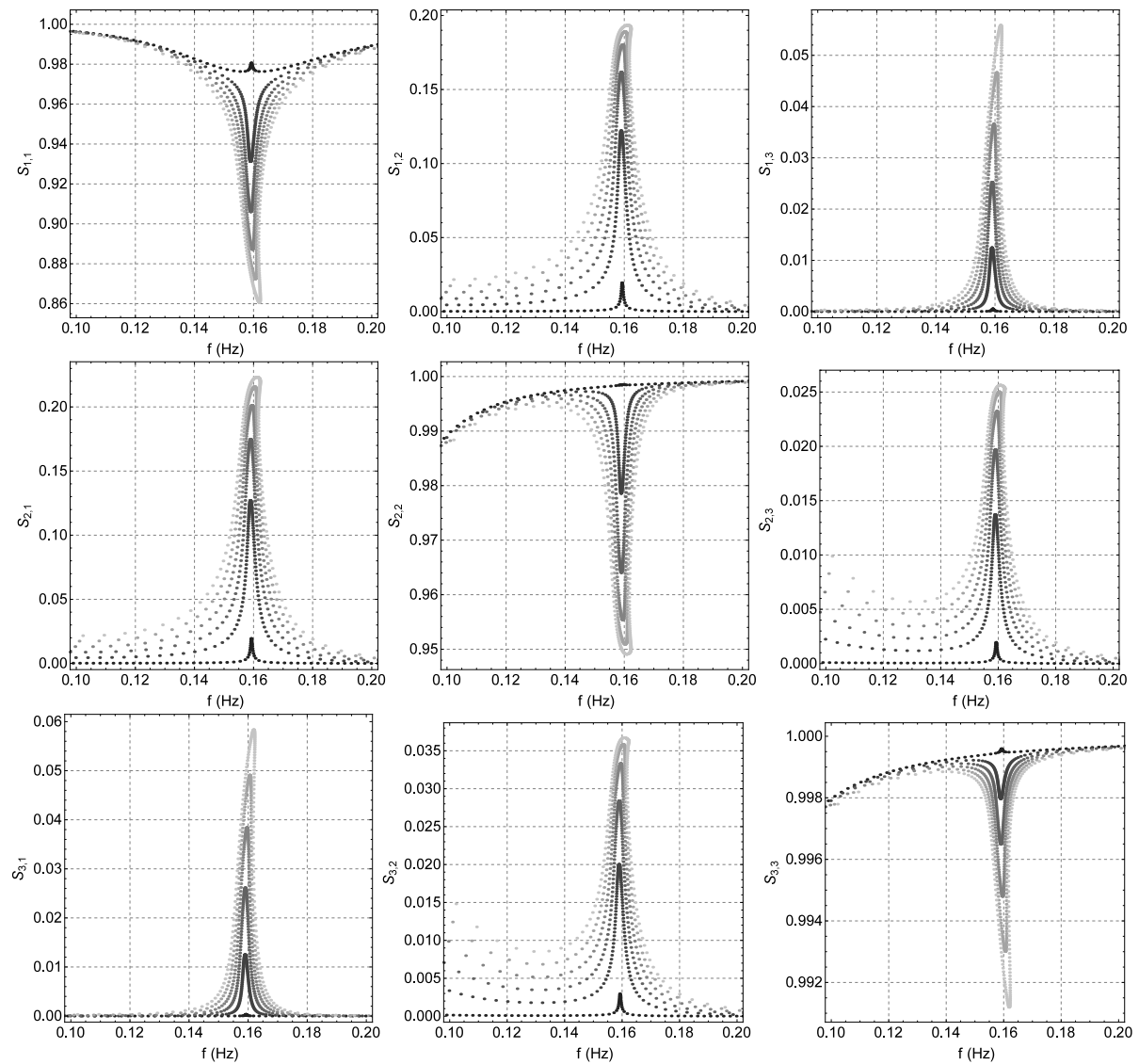

Figure 11: Modulus of the components of the matrix $\mathbf{S}$ obtained with the procedure described Section 3.3 for six levels of the fundamental component of $p(t):\left|P_{1}\right|=0.01,0.1,1,10,50$, and 100. (displayed in gray level from bottom to top).

of $\mathbf{P}^{-}$obtained from Eq. 54 neglecting the matrix $\mathbf{T}$ and reducing $\mathbf{S}$ to this first column and to the linear part of the diagonal terms. To be more specific, $S_{2,2}$ and $S_{3,3}$ were obtained from Eq. (56) using the part of the Jacobian $\mathbf{J}_{\mathbf{F}}(0)$ due to the linear part of $\mathbf{F}$. The results show a good agreement with both the HBM approach and the prediction including all the structure of $\mathbf{S}$. 

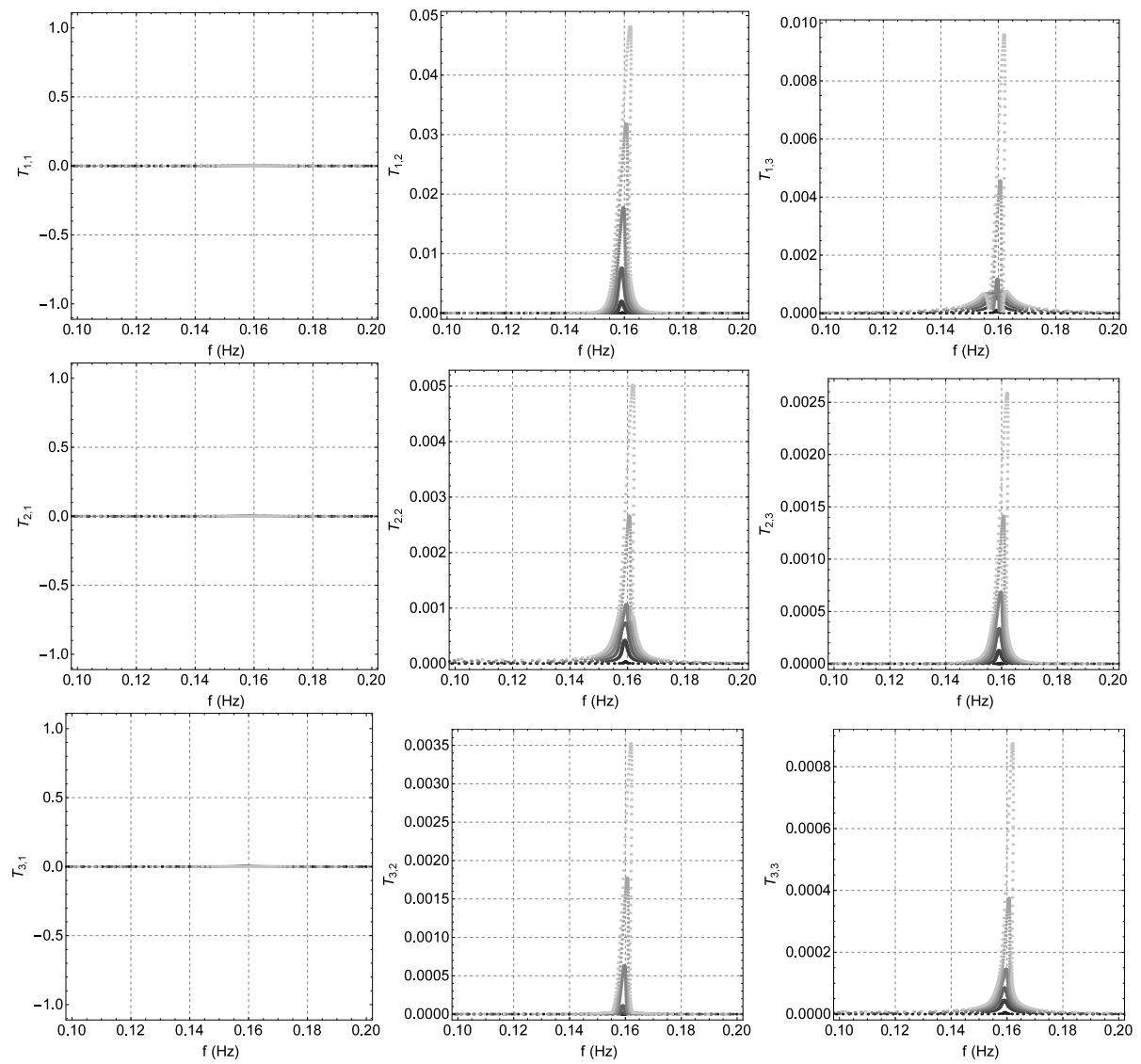

Figure 12: Modulus of the components of the matrix $\mathbf{T}$ obtained with the procedure described Section 3.3 for six levels of the fundamental component of $p(t):\left|P_{1}\right|=0.01,0.1,1 ., 10$. and 100. (displayed in gray level from bottom to top).

\section{Conclusion}

In this work we study energy transfer in the one-port nonlinear class of acoustical systems. Our results apply to nonlinear systems submitted to a tonal (mostly monochromatic) harmonic excitation.

We establish the formulation of the scattering matrices $\mathbf{S}$ and $\mathbf{T}$ analytically from the dynamical description (in our case as differential equations) of the nonlinear system and the harmonic balance approximations. The scattering matrices describe the response of the nonlinear system in terms of the harmonics 

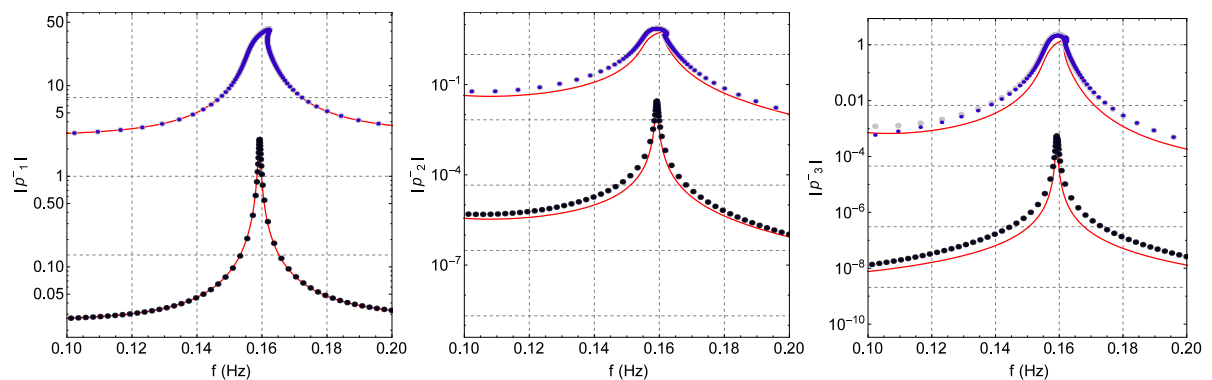

Figure 13: Modulus of the harmonic components of $p^{-}(t)$ obtained with the HBM (continuous red lines) and with the polyharmonic distortion decomposition Eq. 54 taking into account only the matrix $\mathbf{S}$ (dots displayed in gray level) and taking into account only the reduced matrix $S$ (blue dots) for two levels: $\left|P_{1}\right|=0.1$ and 100 .

of the backward acoustic wave with respect to the harmonics of the incoming acoustic wave. We notice that for systems which nonlinearity is holomorphic, the matrix $\mathbf{T}$ is null.

We apply these results to two examples of nonlinear, dynamic absorbers. The first one is a membrane which first mode exhibits large deformations, with a cubic term in the relationship between pressure and displacement (i.e. in the equation of motion). The second one is a Helmholtz resonator with quadratic and cubic terms in the relationship between pressure and displacement. For both cases, The matrices give polyharmonic responses of the devices almost identical to calculation made with numerical, time integration of the equations of motion. In our examples the matrix $\mathbf{T}$ is not null, even though its coefficients are small with respect to the highest coefficients of $\mathbf{S}$ and could be probably neglected.

The highest coefficients of $\mathbf{S}$ are the diagonal coefficients, which means that the energy of the backward wave is mostly in its fundamental component. A further physical interpretation can be done : the energy lost by the fundamental 475 inward wave when it is reflected on the absorber, is higher than the sum of the energy diffused backward by the higher harmonics, which is consistent with the passive nature of the absorbers. This is consistent with small off-diagonal coefficients. We stress the fact that the same system could be described with 
the same accuracy by other matrices, even some with off-diagonal terms much higher than the diagonal terms, or some full matrices. This implies probably to set explicit rules for the construction of the diffusion matrices in view of standardization or physical interpretation.

We have been developing a setup for the nonlinear characterization of acoustic absorbers [12] that may measure the coefficients of the scattering matrices $\mathbf{S}$ and $\mathbf{T}$. This study will be useful for modeling the responses of known nonlinear systems in order to compare experimental and numerical results.

In this work, the scattering matrices are established for a wide range of systems and may be applied to nonlinear systems in other fields of physics, like those submitted to electromagnetic waves.

\section{Acknowledgment}

This work was made in the wireframe of the LABEX MEC and Initiative d'Excellence A*MIDEX.

\section{Appendix A. Continuation procedure}

An arc-length continuation method is used to solve Eq. 62 considering ${ }_{495} \quad \mathbf{V V}^{\mathrm{RI}}$ and $f$ as unknown.

Starting from a root $\left(\overline{\mathbf{V V}}^{\mathrm{RI}}, \bar{f}\right)$ of

$$
\tilde{\mathbf{R}}^{\mathrm{RI}}\left(\bar{f}, \mathbf{V V}^{\mathrm{RI}}, \mathbf{P}^{\mathrm{RI}}(\bar{f})\right)=0
$$

a solution path with respect to the length variable $s$ is obtained solving the system of Algebraic-Differential Equations (ADE)

$$
\begin{gathered}
\sum_{m=1}^{2 H+1}\left(\frac{d \mathbf{V} \mathbf{V}_{m}^{\mathrm{RI}}}{d s}(s)\right)^{2}+\left(\frac{d f}{d s}(s)\right)^{2}=1, \quad \text { for } s_{1}<s<s_{2} \\
\tilde{\mathbf{R}}^{\mathrm{RI}}\left(f(s), \mathbf{V} \mathbf{V}^{\mathrm{RI}}(s), \mathbf{P}^{\mathrm{RI}}(f(s))\right)=0, \quad \text { for } s_{1}<s<s_{2}
\end{gathered}
$$


associated to the initial conditions

$$
\begin{aligned}
f(0) & =\bar{f}, & \frac{d f}{d s}(0) & =1, \\
\mathbf{V V}_{m}(0) & =\overline{\mathbf{V V}}_{m}^{\mathrm{RI}}, & \frac{d \mathbf{V} \mathbf{V}_{m}^{\mathrm{RI}}}{d s}(0) & =0, \quad \text { for } m=1, \ldots, 2 H+1 .
\end{aligned}
$$

The ADE A.2a-A.3b are solved using NDSolve whereas the root A.1 is obtained with FindRoot available in (c) Mathematica.

\section{Appendix B. The model parameters of the NES}

As established in [7, the parameters of the NES model (66) are given by

$$
m_{\mathrm{m}}=\rho_{\mathrm{m}} h_{\mathrm{m}} S_{\mathrm{m}}, k_{\mathrm{m}}=k_{0 \mathrm{~m}} \frac{f_{1 \mathrm{~m}}^{2}}{f_{0 \mathrm{~m}}^{2}}, c_{\mathrm{m}}=\eta k_{0 \mathrm{~m}} \text { and } \quad k_{3 \mathrm{~m}}=\frac{1}{2} \frac{8 \pi E_{\mathrm{m}} h_{\mathrm{m}}}{3\left(1-\nu^{2}\right) R_{\mathrm{m}}^{2}}
$$

with

$$
k_{0 \mathrm{~m}}=\frac{2 \pi E h_{\mathrm{m}}^{3}}{3\left(1-\nu^{2}\right) R_{\mathrm{m}}^{2}} \quad \text { and } \quad f_{0 \mathrm{~m}}=\frac{1}{2 \pi} \sqrt{\frac{1.015^{4} \pi^{4} E h_{\mathrm{m}}^{2}}{12\left(1-\nu^{2}\right) \rho_{\mathrm{m}} R_{\mathrm{m}}^{4}}}
$$

where $\rho_{\mathrm{m}}$ is the mass volume, $\nu$ the Poisson's coefficient and $E$ the Young's modulus of the membrane (made here in latex), $h_{\mathrm{m}}$ is its thickness, $R_{\mathrm{m}}$ its radius and $S_{\mathrm{m}}$ its area. The coefficients $k_{1 \mathrm{~m}}$ and $k_{3 \mathrm{~m}}$ stand for the linear and nonlinear stiffness coefficients, respectively. As suggested and justified in [7, the coefficient $k_{3 \mathrm{~m}}$ was divided by 2 [19]. $f_{0 \mathrm{~m}}$ represents the resonance frequency of the membrane without pre-stress. The last two parameters $f_{1 \mathrm{~m}}\left(\geq f_{0 \mathrm{~m}}\right)$ and $\eta(>0)$ characterizes the pre-tension applied to the membrane and the damping. When no pre-tension is considered, $f_{1 \mathrm{~m}}=f_{0 \mathrm{~m}}$.

The NES membrane under study is defined from the numerical values of the parameters given in terms of geometrical quantities $R_{\mathrm{m}}=0.03 \mathrm{~m}, S_{\mathrm{m}}=$ $\pi 0.03^{2} \mathrm{~m}^{2}, h_{\mathrm{m}}=0.18 \times 0.001 \mathrm{~m}$; in terms of material quantities $\rho_{\mathrm{a}}=1.17 \mathrm{~kg} \mathrm{~m}^{-3}$, $c_{\mathrm{a}}=344 \mathrm{~m} \mathrm{~s}^{-1}$ for the air and $\rho_{\mathrm{m}}=980 \mathrm{~kg} \mathrm{~m}^{-3}, E=1200000 \mathrm{~Pa}$ and $\nu=0.49$ for latex membrane, and, in terms of damping quantities $\eta=0.00114$. With these parameters the resulting resonance frequency of the linear part of membrane is $f_{0 \mathrm{~m}}=3.45 \mathrm{~Hz}$. Finally, we select the pre-tension parameter as $f_{1 \mathrm{~m}}=40 \mathrm{~Hz}$. Note that the values of the parameters related to the membrane 
$\left(R_{\mathrm{m}}, h_{\mathrm{m}}, \rho_{\mathrm{m}}, E, \nu, \eta\right.$ and $\left.f_{1 \mathrm{~m}}\right)$ have been chosen following the recommendations discussed in [7] in reference to the experiment data.

\section{Compliance with Ethical Standards} concerning the publication of this manuscript.

\section{References}

[1] A. Vakakis, O. Gendelman, L. Bergman, D. McFarland, G. Kerschen, Y. Lee, Nonlinear targeted energy transfer in mechanical and structural systems, Vol. 156 of Solid mechanics and its applications, Springer, Berlin, 2008.

[2] E. Gourdon, N. Alexander, C. Taylor, C.-H. Lamarque, S. Pernot, Nonlinear energy pumping under transient forcing with strongly nonlinear coupling: Theoretical and experimental results, J Sound Vib. 300 (2007) 522551. doi:https://doi.org/10.1016/j.jsv.2006.06.074

[3] P.-O. Mattei, R. Ponçot, M. Pachebat, R. Côte, Nonlinear targeted energy transfer of two coupled cantilever beams coupled to a bistable light

a attachment, J Sound Vib. 373 (Supplement C) (2016) 29-51. doi:https: //doi.org/10.1016/j.jsv.2016.03.008

[4] G. Sigalov, O. Gendelman, M. AL-Shudeifat, L. Manevitch, A. Vakakis, L. Bergman, Resonance captures and targeted energy transfers in an inertially-coupled rotational nonlinear energy sink, Nonlinear Dyn 69 (2012) 1693-1704. doi:https://doi.org/10.1007/s11071-012-0379-1.

[5] E. Gourc, S. Seguy, G. Michon, A. Berlioz, B. Manne, Quenching chatter 540 instability in turning process with a vibro-impact nonlinear energy sink, J Sound Vib. 355 (2015) 392-406. doi:https://doi.org/10.1016/j.jsv. 2015.06 .025 
[6] B. Cochelin, P. Herzog, P.-O. Mattei, Experimental evidence of energy pumping in acoustics, C. R. Mécanique 334 (11) (2006) 639-644.

[7] R. Bellet, B. Cochelin, P. Herzog, P.-O. Mattei, Experimental study of targeted energy transfer from an acoustic system to a nonlinear membrane absorber, J Sound Vib. 329 (2010) 2768-2791. doi:https://doi.org/10. $1016 / \mathrm{j} \cdot \mathrm{jsv} .2010 .01 .029$.

[8] R. Mariani, S. Bellizzi, B. Cochelin, P. Herzog, P.-O. Mattei, Toward an adjustable nonlinear low frequency acoustic absorber, J Sound Vib. 330 (2011) 5245-5258. doi:https://doi.org/10.1016/j.jsv.2011.03.034.

[9] P.-Y. Bryk, S. Bellizzi, R. Côte, Experimental study of a hybrid electroacoustic nonlinear membrane absorber, J Sound Vib. 424 (2018) 224-237. doi:https://doi.org/10.1016/j.jsv.2018.03.014.

[10] E. Gourdon, A. T. Savadkoohi, V. A. Vargas, Targeted energy transfer from one acoustical mode to an helmholtz resonator with nonlinear behavior, J Vib Acoust 140 (2018). doi:https://doi.org/10.1115/1.4039960.

[11] J. Dalmont, Acoustic impedance measurement. part i: a review, J Sound Vib. 3 (2001) 427-439. doi:https://doi.org/10.1006/jsvi.2000.3428.

[12] A. Chauvin, M. Monteil, S. Bellizzi, R. Côte, P. Herzog, M. Pachebat, Acoustic characterization of a nonlinear vibroacoustic absorber at low frequencies and high sound levels, J Sound Vib. 416 (2017) 244-257. doi:https://doi.org/10.1016/j.jsv.2017.11.031.

[13] J. Verspecht, D. E. Root, Polyharmonic distortion modeling, IEEE Mi565 crowave Magazine 7 (3) (2006) 44-57. doi:https://doi.org/10.1109/ MMW.2006.1638289.

[14] H. Bodén, One-sided multi-port techniques for characterisation of in-duct samples with nonlinear acoustic properties, J Sound Vib. 331 (2012) 30503067. doi:https://doi.org/10.1016/j.jsv.2012.02.013 
[15] M. Monteil, O. Thomas, C. Touzé, Identifcation of mode couplings in nonlinear vibrations of the steelpan, Appl Acoust 89 (2015) 1-15. doi:https: //doi.org/10.1016/j.apacoust.2014.08.008.

[16] M. Krack, J. Gross, Harmonic bamance for nonlinear vibration problem, Springer, 2019.

[17] V. Alamo Vargas, E. Gourdon, A. Ture Savadkoohi, Nonlinear softening and hardening behavior in helmholtz resonators for nonlinear regimes,

■ Nonlinear Dyn 91 (2018) 217-231. doi:https://doi.org/10.1007/ s11071-017-3864-8.

[18] V. A. Vargas, Transfert énergétique irréversible grâce à un résonateur acoustique à comportement non-linéaire [irreversible energy transfer using an acoustic resonator with a nonlinear behavior], Ph.D. thesis, Université de Lyon - ENTPE (2018).

[19] R. Bellet, B. Cochelin, R. Côte, P.-O. Mattei, Enhancing the dynamic range of targeted energy transfer in acoustics using several nonlinear membrane absorbers, J Sound Vib. 331 (26) (2012) 5657-5668. doi:https://doi. org $/ 10.1016 / j \cdot j s v .2012 .07 .013$. 